\title{
Polymorphisms within the COL5A1 gene and regulators of the extracellular matrixmodify the risk of Achilles tendon pathology in a British case-control study
}

Karryn L Brown; Kirsten B Seale; Louis Y. El Khoury, Michael Posthumus; William J Ribbans; Stuart M Raleigh; Malcolm Collins; Alison V September.

\section{Abstract}

Several genetic loci have been associated with risk of Achilles tendon pathology (ATP) within South African and Australian populations. The aim of this study was, therefore, to evaluate eight previously implicated genetic variants in an independent British population. A total of 130 asymptomatic controls (CON) and 112 participants clinically diagnosed with ATP comprising 87 individualswith chronic Achilles tendinopathy (TEN) and 25 with Achilles tendon ruptures (RUP) were included. All participants were genotypedfor variants within the COL5A1, MIR608, IL-1 $\beta, I L-6$ and CASP8 genes. Primary findings implicated COL5A1 and CASP8. Three inferredallele combinations constructed from COL5A1 rs 12722 , rs3196378 and rs71746744 were identified as risk modifiers. The T-C-D combination was associated with increased risk of ATP $(P=0.023)$ and RUP $(P<0.001)$, the $\mathrm{C}-\mathrm{A}-\mathrm{I}$ combination was associated with increased risk of ATP $(P=0.011)$, TEN $(P=0.011)$ and RUP $(P=0.011)$ and the C-C-D combination was associated with decreased riskof ATP $(P=0.011)$ and RUP $(P=0.004)$. The CASP8 rs3834129 DD genotype was associated with decreased risk of TEN $(P=0.020$, odds ratio: $0.45,95 \%$ confidence interval: 0.22-0.90) and the CASP8 I-G (rs3834129-rs1045485) inferred allele combination was associated with increased risk of TEN $(P=0.031)$. This study further highlights the importance of polymorphisms within COL5A1 and CASP8 inthe aetiology of ATP.

\section{Introduction}

Achilles tendon pathology (ATP) is an encompassing term describing both chronic Achilles tendinopathy (TEN) and acute Achilles tendon ruptures (RUPs), which are common among athletes (reviewed in Järvinen, Kannus, Maffulli, \& Khan, 2005). Both pathologies are multifactorial and influenced by both extrinsic and intrinsic risk factors, where an individual's genetic profile has been identified as one of several intrinsic risk factors (Collins, September, \& Posthumus, 2015; Meeuwisse, 1994).

Several loci have been characterised in the aetiology of ATP based on the hypothesis that sequence variants may alter the tendon extracellular matrix (ECM) structure and/or regulation, thereby modifying ATP susceptibility (reviewed in Collins et al., 2015). Implicated genes include: COL5A1, which encodes the $\alpha 1$ chains of the heterotrimeric type $\mathrm{V}$ collagen and regulates collagen fibrillogenesis (Wenstrup et al., 2004); MIR608, which encodes, the micro-RNA, Hsa-miR-608 and may influence fibrillogenesis via its post-transcriptional regulation of COL5A1 and other ECM mRNAs (Abrahams, Laguette, Prince, \& Collins, 2013); and the ECM regulators IL-1B, IL-6 and CASP8 which encode interleukin-1B (IL-1 $\beta$ ), interleukin-6 (IL-6), and caspase-8 (CASP-8) and operate in overlapping cell signalling and apoptosis pathways (Nell et al., 2012; September et al., 2011) with the aim to maintain ECM homeostasis in response to mechanical stimuli (Riley, 2005; Yuan, Murrell, Wei, \& Wang, 2002).

These genes function collectively in interdependent relationships thereby potentially influencing the balance between synthesis and degradation of the components of the ECM of tendons. Figure 1 is a summary highlighting some of the proposed biological relationships between these ECM components in mechanically loaded tendons (September et al., 2011). In the event of mechanical loading, cell-signalling molecules such as IL-1 $\beta$ and IL-6 are up-regulated (Tsuzaki et al., 2003). Cytokines such as the interleukins form part of the inflammatory response (Tsuzaki et al., 2003), and it has been noted that early inflammation potentially plays a role in the aetiology of ATP (Scott, Backman, \& Speed, 2015). Increased adiposity has been highlighted as a risk factor for ATP (Gaida, Ashe, Bass, \& Cook, 2009) and evidence is mounting to suggest that a mechanism for this may be due to increased adiposity producing higher levels of circulating interleukins (Fain, 2006). Moreover, there is evidence to suggest that IL-1 and IL- 6 variants, including IL-6 rs 1800795 have been implicated in the regulation of general adiposity (Strandberg et al., 2008). 
apoptotic signalling cascade (Millar, Wei, Molloy, Bonar, \& Murrell, 2009; Nell et al., 2012). This is a normal healing process (Nell et al., 2012) and the cell's defence mechanism when tendons experience mechanical strain (Millar et al., 2009; Nell et al., 2012). In addition to these apoptotic mediators, matrix degrading enzymes (Sun et al., 2008; Thampattya, Li, Im, \& Wang, 2007; Tsuzaki et al., 2003) and molecules, which alter COL5A1 expression are stimulated (Kahai, Vary, Gao, \& Seth, 2004; Villiger, Kusari, ten Dijke, \& Lotz, 1993). The downstream effects culminate in tenocyte apoptosis (Nell et al., 2012), type $V$ collagen degradation (Birkedal-Hansen et al., 1993) and increased COL5A1 gene expression, which collectively influence tendon capacity and most likely ATP susceptibility (Nell et al., 2012). Identification of genetic sequence variants, which influence the regulation of the synthesis and degradation of the components of the ECM of tendons, could assist in elucidating the aetiology of ATP.

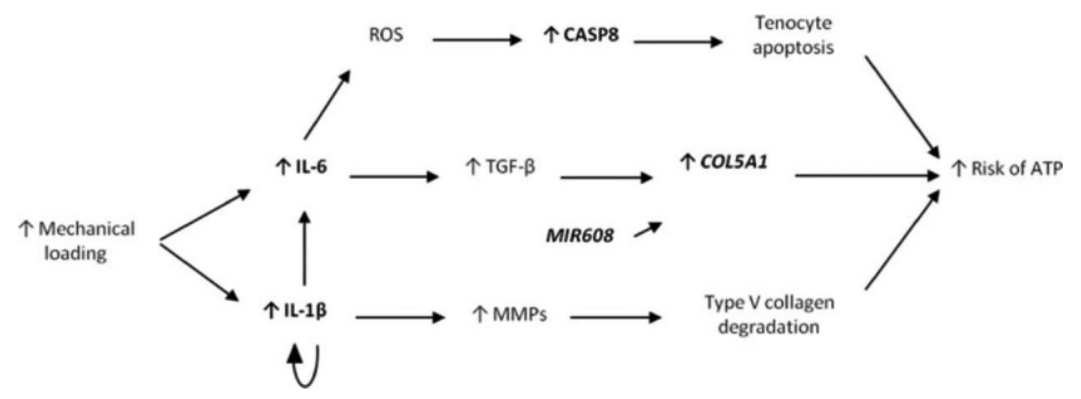

Figure 1. Summary of some of the interdependent relationships between ECM components in mechanically loaded tendons and their potential effect on the risk of ATP development. IL- $1 \beta$ and IL- 6 are induced by increased mechanical loading (Nell et al., 2012; Tsuzaki et al., 2003). IL-1 $\beta$ further up-regulates its own expression and activates MMPs, resulting in ECM degradation (Sun et al., 2008; Thampattya et al., 2007; Tsuzaki et al., 2003), including that of type $\mathrm{V}$ collagen(Birkedal-Hansen et al., 1993). IL-6 which is further up-regulated by IL-1 $\beta$ (Tsuzaki et al., 2003) can increase COL5A1 gene expression but primarily operates in the apoptosis signalling pathway. In this pathway, increased IL-6 expression further induces the production of ROS resulting in synthesis of the apoptotic mediator CASP-8 (Millar et al., 2009; September et al., 2011) and increased tenocyte apoptosis (Nell et al., 2012). Type $V$ collagen degradation, increased COL5A1 gene expression and tenocyte apoptosis may all increase the risk of ATP (September et al., 2011). MIR608 which regulates type $V$ collagen production (Abrahams et al., 2013) is also shown. The molecules and genes investigated in this study are shown in bold. The upwards arrows indicate increased expression. IL-1 $\beta$ : interleukin-1 $\beta$, IL-6 interleukin-6, MMPs: matrix metalloproteinases, TGF- $\beta$ : transforming growth factor $\beta$, ROS: reactive oxygen species, CASP8: caspase8, ATP: Achilles tendon pathology.

Several sequence variants within COL5A1 (Mokone, Schwellnus, Noakes, \& Collins, 2006; September et al., 2009), MIR608 (Abrahams et al., 2013), IL-1B, IL-6 (September et al., 2011) and CASP8 (Nell et al., 2012) have been explored in South African and Australian study participants and require further validation in independent populations (Chanock et al., 2007; Collins et al., 2015). The aims of this study were therefore to evaluate COL5A1 rs12722 (C/T), COL5A1 rs3196378 (C/ A), COL5A1 rs71746744 (insertion/deletion), MIR608 rs4919510 (C/G), IL-1ß rs16944 (C/T), IL-6 rs1800795 (G/C) as well as CASP8 rs1045485 (G/C) and CASP8 rs3834129 (insertion/deletion) for independent and collective associations with ATP in a British cohort.

The a priori hypothesis was that (i) the COL5A1 rs3196378 C allele (September et al., 2009) the COL5A1 rs71746744 insertion allele (Abrahams et al., 2013), the MIR608 rs4919510 CC genotype (Abrahams et al., 2013), the IL-1 $\beta$ rs16944 C allele (September et al., 2011) and the IL-6 rs1800795 G allele (September et al., 2011) would be associated with an increased of risk of ATP, (ii) the COL5A1 rs12722 CC genotype (September et al., 2009), the CASP8 rs1045485 C allele (Nell et al., 2012) and the CASP8 rs3834129 deletion allele (Nell et al., 2012) would be associated with a decreased risk of ATP and (iii) that the following inferred allele combinations would be associated with an increased risk of ATP: T-C-I (COL5A1: rs12722rs3196378-rs71746744) (Abrahams et al., 2013; September et al., 2009); A-C (COL5A1 rs3196378- 
MIR608 rs4919510) (Abrahams et al., 2013); C-G-T (IL-1ß

rs16944-IL-6 rs1800795-COL5A1 rs12722) (September et al., 2011) and I-G (CASP8: rs3834129rs1045485) (Nell et al., 2012).

\section{Methods}

\section{Participants}

A case-control genetic association study was employed, and the reporting of this study is in accordance with the STREGA initiative recommendations (Little et al., 2009); an extension of the STROBE statement (Von Elm et al., 2007). During the period of June 2011 to June 2014, 267 British participants were recruited. The participants consisted of a control group (CON group) comprising 130 apparently healthy individuals and an ATP group ( $n=112$ ) comprising of 87 and 25 individuals diagnosed with (TEN sub-group) and (RUP subgroup) respectively

These participants were previously recruited and described by Rickaby et al. (Rickaby, El Khoury, Ribbans, \& Raleigh, 2015). All participants in the CON group and the ATP group were recruited from the same geographical area within the East Midlands region of the United Kingdom. In addition, the CON and ATP groups were matched for age and level of physical activity, at recruitment. After recruitment, 25 individuals were excluded based on the inclusion and exclusion criteria described by Mokone et al. $(2005,2006)$. Participants who had used fluoroquinolone antibiotics (two exclusions) or local corticosteroid injections (two exclusions) into the Achilles tendon or surrounding structures prior to experiencing symptoms were excluded. This was necessary as fluoroquinolone antibiotics and corticosteroids increase the risk of RUPs (Newnham, Douglas, Legge, \& Friend, 1991; Van der Linden et al., 2001). In addition, participants diagnosed with connective tissue disorders or systemic diseases possibly linked to ATP were excluded (15 exclusions) (Mokone et al., 2006) together with 3 non-Caucasian

participants and 3 participants originally recruited for the CON group who subsequently developed an injury. The TEN sub-group experienced gradual progressive pain in the area of the Achilles tendon for more than 6 months accompanied by at least one of the following Achilles tendon symptoms: (1) early morning pain, (2) early morning stiffness,

(3) swelling, (4) tenderness to palpation, (5) palpable nodular thickening or (6) movement of the painful region with plantardorsi-flexion (positive "shift" test) (Mokone et al., 2005, 2006). Individuals in the TEN subgroup were diagnosed with insertional $(n=25)$ or non-insertional tendinopathy $(n=48)$ and in addition, some individuals presented with both insertional and non-insertional tendinopathies $(n=14)$. All clinical diagnoses of tendinopathy were confirmed by an ultrasound or magnetic resonance imaging (MRI). Individuals in the RUP sub-group were diagnosed with complete $(n=18)$ or partial ruptures $(n=7)$. All clinical diagnoses of ruptures were confirmed at the time of surgery or by imaging. Nine individuals who sustained RUPs were also diagnosed with tendinopathy but for the purpose of analyses were included in the RUP sub-group.

All of the participants were of self-reported British Caucasian ancestry and signed written informed consent according to the Declaration of Helsinki. Questionnaires regarding physical activity details, injury details and medical history were also completed by each participant. Ethical approval for this study was obtained from the Human Research Ethics Committee of the Faculty of Health Sciences, University of Cape Town (reference number 370/2013) and the Research Ethics Committee of the University of Northampton.

\section{DNA extraction}

Approximately $2 \mathrm{ml}$ of saliva was collected from each participant for the DNA extraction as previously described, using the ORAGENE DNA kits (OG-500) and the prepIT-L2P DNA extraction kit (DNA Genotek Inc., ON, Canada), which yielded A260/ 280 ratios between: 1.8 and 2.0 (Rickaby et al., 2015). All DNA extractions were conducted at the University of Northampton and made available for this study.

Polymorphism analysis and genotyping

All eight polymorphisms investigated in this study were previously implicated in ATP (Abrahams et al., 2013; Nell et al., 2012; September et al., 2009, 2011) and their minor allele frequencies are noted in supplementary Table 1. Polymorphisms included three single nucleotide polymorphisms (SNPs) and a short tandem repeat (STR) within two genes proposed to influence collagen fibril formation [COL5A1 rs12722 
(C/T), COL5A1 rs71746744 (insertion/deletion), COL5A1 rs3196378 (C/A), and MIR608 rs4919510 (C/G)] as

well as three SNPs and a STR within three genes involved in the cell-signalling and apoptosis pathways [IL$1 \beta$ rs16944 (C/T), IL-6 rs1800795 (G/C), CASP8 rs1045485 (G/C) and CASP8

rs3834129 (insertion/deletion)]. Moreover, the specific polymorphisms selected have been implicated to be of functional significance or have been localized to a region that is of functional significance in the aetiology of ATP (Abrahams et al., 2013; Nell et al., 2012; September et al., 2009, 2011).

All participants were genotyped for these polymorphisms using one of two methods. The TaqManTM allelic discrimination assay (Applied Biosystems, Foster City, CA, USA) was used to genotype COL5A1 rs3196378, COL5A1 rs71746744, MIR608

rs4919510, CASP8 rs1045485 and CASP8 rs3834129. In this case the Applied Biosystems StepOnePlus ${ }^{\mathrm{TM}}$ Real-Time PCR System (Applied Biosystems, Foster City, CA, USA) and the Applied Biosystems StepOnePlus ${ }^{\text {TM }}$ Real-Time PCR software v2.2.2. (Applied Biosystems, Foster City, CA, USA) were used for polymerase chain reaction (PCR) reactions and genotype determination according to manufacturer's recommendations. Standard PCR-based restriction fragment length polymorphism (RFLP) analysis was used to genotype COL5A1 rs12722 (BstUI), IL-1ß rs16944 (Aval) and IL-6 rs1800795 (NlallI) as previously described (Mokone et al., 2006; September et al., 2011). After enzymatic digestion of the PCR amplicons, COL5A1 rs 12722 restriction fragments were resolved on $6 \%$ polyacrylamide gels while IL-1 $\beta$ rs 16944 and IL-6 rs 1800795 restriction fragments were resolved on $2 \%$ agarose gels. Digestion of the $667 \mathrm{bp}$ COL5A1 amplicon produced $316 \mathrm{bp}, 271 \mathrm{bp}$ and $80 \mathrm{bp}$ fragments for the $\mathrm{C}$ allele and $351 \mathrm{bp}$ and $316 \mathrm{bp}$ fragments for the T allele. Digestion of the 304bp IL-1 $\beta$ amplicon produced a 304bp fragment for the T allele and 190bp and $114 \mathrm{bp}$ fragments for the $C$ allele. Digestion of the 228bp IL-6 amplicon produced $172 \mathrm{bp}$ and $56 \mathrm{bp}$ fragments for the $G$ allele and $123 \mathrm{bp}, 56 \mathrm{bp}$ and $49 \mathrm{bp}$ fragments for the $C$ allele. All restriction fragments were resolved alongside a 100bp size standard (Promega Corporation, Madison, WI, USA) along with an undigested PCR product, and samples with known genotypes. Products were visualized under ultraviolet light with the SYBR® Gold Nucleic Acid Gel Stain (Invitrogen Molecular Probes, OR, USA). In addition, each PCR plate (TaqManTM and RFLP) contained samples with known genotypes and samples with no DNA to ensure consistent genotyping and detect potential contamination. To avoid genotyping errors, samples that failed twice to amplify during PCR were considered as unsuccessfully genotyped and no further attempts were made to genotype them at that polymorphism locus. Samples that consistently failed to amplify during PCR were excluded in further studies. All genotype analyses were confirmed by two independent researchers and conducted at the Division of Exercise Science and Sports Medicine, Department of Human Biology, University of Cape Town or at the University of Northampton, UK.

Statistical analysis

Statistical power calculations were previously calculated using Quanto v1.2. (http://hydra.usc.edu/gxe) (Rickaby et al., 2015). STATISTICA v12 (StatSoft Inc., Tulsa, OK, USA) was used to compare basic participant characteristics of the study groups using a one way analysis of variance (ANOVA). The programming environment $R$ (R Development Core Team, 2013) and R package, SNPassoc (González et al., 2007), was used to calculate and analyse genotype frequency distributions and for Hardy-Weinberg Equilibrium probabilities while the R package, Haplo.stats, (Sinnwell \& Schaid, 2008) was used to analyse inferred allele combinations. GraphPad Prism v5.02 was used to analyse allele

frequency distributions. All genotype and allele frequency distributions were analysed using Pearson's chisquared analyses $(\mathrm{X} 2)$ or Fisher's exact tests. Regarding genotype frequencies, global P-values were initially analysed followed by P-values for dominant, recessive and over-dominant models if significance or trends towards significance were observed as our study was based on an a priori hypothesis. Similarly, for inferred allele combinations, global P-values for additive, dominant and recessive models were analysed followed by P-values for each allele combination within the different models. In all instances, the lowest $\mathrm{P}$-value indicates the best fit model and these are the $\mathrm{P}$-values which are reported. Significance was accepted at $\mathrm{P}<0.05$ for all analyses. Information regarding basic participant characteristics was not available for all participants; therefore, analyses were conducted without adjusting for covariates and without stratifying by sex to retain the largest possible sample size.

Results 
Participant characteristics

The CON and ATP groups as well as the CON group and RUP sub-group were similarly matched for age, height, weight, BMI, sex and country of birth (Table 1). The CON group and the TEN sub-group were similarly matched for all characteristics except age $(P=0.044)$ where the CON group participants were significantly younger at age of recruitment than the TEN subgroup participants were at age of initial onset of symptoms. There were no significant genotype effects on any of the participant characteristics (data not shown) except for COL5A1 rs12722 (C/T) and IL-6 rs1800795 (G/C), which showed significant effects on BMI $(P=0.027)$ and sex $(P=0.046)$, respectively. On average, individuals with a COL5A1 rs12722 TT genotype had a significantly lower BMI than individuals with a CT genotype $(P=0.019)(T T: 24.7 \pm 4.34$ $\mathrm{kg} / \mathrm{m} 2$; CT:

$26.69 \pm 4.25 \mathrm{~kg} / \mathrm{m} 2)$ and in addition there were significantly more males than females with the IL-6 rs1800795 GG genotype ( $P=0.014)$. Both the CON and ATP groups were similarly grouped for levels of vigorous physical activity as defined by Haskell et al.(Haskell et al., 2007). Mean values (hours/week) were $4.46 \pm 4.24$ for the CON group and $4.19 \pm 3.73$ for the ATP group. No significant differences $(P>0.05)$ between these values were noted.

Genotype and allele frequencies

There were no independent significant differences in the genotype or allele frequency distributions between any of the groups for the three COL5A1 variants (Table 2). MIR608 rs4919510 and IL-1B rs16944 showed no significant differences in genotype or allele frequency distributions between the CON and ATP groups or between the CON group and TEN subgroup. There were, however, significant differences in genotype distributions between the CON group and the RUP subgroup (Table 2). The CG genotype of MIR608 rs4919510 was significantly associated with a decreased risk of RUP (overdominant model: $P=0.022$, odds ratio [OR]: $0.30,95 \%$ confidence interval $[C I]$ : 0.10-0.93). The CT genotype of IL-1 $\beta$ rs 16944 was significantly associated with an increased risk of RUP (over-dominant model: $\mathrm{P}=0.012$, OR: $3.96,95 \% \mathrm{Cl}$ : 1.24- 12.70). These findings should, however, be interpreted with caution owing to the sample size limitation of the RUP subgroup. IL-6 rs1800795 and CASP8 rs1045485 showed no significant differences in genotype or allele frequency distributions between any of the groups (Table 2). CASP8 rs3834129 also showed no significant differences in genotype or allele frequencies but a trend suggesting differing genotype distributions between the CON group and TEN sub-group was observed $(P=0.060)$. Further analyses following the a priori hypothesis indicated that the DD genotype was significantly associated with a decreased risk of TEN (recessive model: $\mathrm{P}=0.020$, OR: $0.45,95 \% \mathrm{Cl}: 0.22-0.90)$. The genotype distributions of all the groups were in Hardy-Weinberg equilibrium except for the RUP sub-groups of COL5A1 rs12722 and IL-1 $\beta$ rs16944 (Table 2).

Inferred allele combinations Inferred allele combinations were constructed from COL5A1 rs12722, rs3196378 and rs71746744 with six possible combinations noted [Figure 2(a)]. The T-A-I inferred allele combination was the most common (overall frequency: $45 \%$ ) while the T-C-I and T-C-D inferred allele combinations were the least common (overall frequency: $3 \%$ ). Global P-values revealed that the COL5A1 inferred allele combinations associated with the risk of ATP (additive and dominant models: $P=0.003$ ) and RUP (additive and dominant models: $P$ $<0.001$ ). It was interesting to note that global $P$-values approached significance when the CON group and TEN sub-group was compared (dominant

Table 1. Participant characteristics of the control group (CON), Achilles tendon pathology group (ATP), Achilles tendinopathy sub-group (TEN) and Achilles tendon rupture sub-group (RUP).

\begin{tabular}{|c|c|c|c|c|c|c|c|}
\hline & $\begin{array}{l}\text { CON }(n= \\
130)\end{array}$ & $\begin{array}{c}\operatorname{ATP}(n= \\
112)\end{array}$ & $\begin{array}{c}P- \\
\text { value } \\
a\end{array}$ & $\begin{array}{l}\text { TEN }(n= \\
87)\end{array}$ & $\begin{array}{c}P- \\
\text { value } \\
\mathrm{b}\end{array}$ & $\begin{array}{c}\operatorname{RUP}(n= \\
25)\end{array}$ & $\begin{array}{c}P- \\
\text { value } \\
c\end{array}$ \\
\hline Age (years) & $41.6 \pm 11.6$ & $43.9 \pm 13.8$ & 0.167 & $45.2 \pm 14.4$ & 0.044 & $39.2 \pm 10.4$ & 0.34 \\
\hline Helght (cm) & $1 / 4.9 \pm 104$ & $1 /(10 \pm 9) y .2$ & U.101 & $1 / 2.3 \pm(8.5)$ & U.US/ & & U. 43 \\
\hline vvelgnt (kg) & ชU. $\angle \pm(124)$ & $\left.11.1 \frac{1}{82}\right)^{10.0}$ & $0.3<4$ & $11.1 \pm 162$ & U. $\angle b 3$ & $19.9(20)^{15.4}$ & $u \cdot y^{1}$ \\
\hline BMI (kg/m) & $25.9 \pm 4.5$ & $26.0 \pm 4.0$ & 0.811 & $25.9 \pm 4.1$ & 0.983 & $26.4 \pm 3.9$ & 0.68 \\
\hline
\end{tabular}




\begin{tabular}{|c|c|c|c|c|c|c|c|}
\hline sex (\%male) & $\begin{array}{l}(123) \\
63.1(82)\end{array}$ & $\begin{array}{c}(8.2) \\
6 \cup .1(68)\end{array}$ & U. IUb & $\begin{array}{c}(62) \\
58.6(51)\end{array}$ & U.SUY & 68.U(1) & $\begin{array}{c}4 \\
\text { U.63 }\end{array}$ \\
\hline $\begin{array}{l}\text { Country ot birth } \\
(\% \cup K)\end{array}$ & $96.0(119)$ & 94.6 (105) & 0.619 & 95.4 (83) & 0.840 & $88.0(22)$ & 0.10 \\
\hline
\end{tabular}

Note: Age, weight and BMI values are self-reported values at the time of recruitment for the CON group and at initial onset of symptoms for the ATP group and the TEN and RUP sub-groups. All values for age, height, weight and $\mathrm{BMI}$ are expressed as the mean \pm the standard deviation while values for sex and country of birth are expressed as percentages. Numbers in parenthesis indicate the number of participants $(n)$. Values in bold typeset indicate significant differences $(P<0.05)$.

${ }^{\mathrm{a} C O N}$ vs.

ATP.

${ }^{\mathrm{b}} \mathrm{CON}$ vs.

TEN.

${ }^{\mathrm{C} C O N}$ vs.

RUP.

Table 2. Genotype and minor allele frequency distributions as well as the calculated Hardy-Weinberg $P$-values for COL5A1 rs12722, COL5A1 rs3196378, COL5A1 rs71746744, MIR608 rs4919510, IL-1 $\beta$ rs16944, IL-6 rS1800795, CASP8 rs1045485 and CASP8 rs3834129 in the control group (CON), Achilies tendon pathology group(ATP), Achilles tendinopathy sub-group (TEN) and Achilles tendon rupture sub-group (RUP).

\begin{tabular}{|c|c|c|c|c|c|c|c|c|}
\hline & & $\begin{array}{c}\mathrm{CO} \\
\mathrm{N}(n= \\
130)\end{array}$ & $\begin{array}{l}\text { ATP } \\
\left(\begin{array}{l}n= \\
112)\end{array}\right.\end{array}$ & $\begin{array}{c}P- \\
\text { value } \\
a\end{array}$ & $\begin{array}{c}\text { TE } \\
\mathrm{N}(n \\
=87)\end{array}$ & $\begin{array}{c}P- \\
\text { value } \\
\mathrm{b}\end{array}$ & $\begin{array}{r}\mathrm{RU} \\
\mathrm{P}(n \\
=25)\end{array}$ & $\underset{c}{P-}$ \\
\hline \multirow{6}{*}{$\begin{array}{l}\mathrm{COL5A1} \text { rs12722 } \\
(\mathrm{C} / \mathrm{T})\end{array}$} & $N$ & 12 & 104 & & 82 & & 22 & \\
\hline & Li & $2 \angle(\angle I)$ & \multirow{5}{*}{$\begin{array}{c}(28) \\
(29) \\
46 \\
(48) \\
(26) \\
4 y \\
(102) \\
0.43\end{array}$} & \multirow[t]{3}{*}{ U.683 } & $(24)$ & \multirow[t]{3}{*}{ U.४५ } & $32(1)$ & \multirow{3}{*}{0.70} \\
\hline & Ui & $51(64)$ & & & 157 & & $21(6)$ & \\
\hline & 11 & $21(34)$ & & & $(24)$ & & $41(y)$ & \\
\hline & \multicolumn{2}{|c|}{ I allele $53(132)$} & & \multirow[t]{2}{*}{0.423} & $(82)$ & \multirow[t]{2}{*}{0.311} & $35(24)$ & \multirow{2}{*}{0.83} \\
\hline & HVVE & U.४Ь४ & & & - & & U.U3y & \\
\hline \multirow{6}{*}{$\begin{array}{l}\text { (CLDA7 rS31963/8 } \\
\text { (C/A) }\end{array}$} & IN & 12 & 108 & & 84 & & 24 & \\
\hline & CL & $26(32)$ & \multirow{5}{*}{$\begin{array}{c}23 \\
(25) \\
45 \\
(49) \\
34 \\
(34) \\
54 \\
(117) \\
0.434\end{array}$} & \multirow[t]{3}{*}{0.119} & (19) & \multirow[t]{3}{*}{0.823} & $25(6)$ & \multirow[t]{3}{*}{ U. 45} \\
\hline & $A C$ & 4 / (๖४) & & & 48 & & $38(y)$ & \\
\hline & $A A$ & $21(33)$ & & & 35 & & $38(y)$ & \\
\hline & \multicolumn{2}{|c|}{ A allele $๖ \cup(124)$} & & \multirow[t]{2}{*}{$U .42 U$} & $(94)$ & \multirow[t]{2}{*}{ U.५ 21} & $56(21)$ & \multirow{2}{*}{$\begin{array}{l}0.4 \zeta \\
9\end{array}$} \\
\hline & HVVE & U.588 & & & 0.66 & & U. 242 & \\
\hline \multirow{6}{*}{$\begin{array}{l}\text { CULSA7 } \\
\text { rs77746744 (D/I) }\end{array}$} & N & $\begin{array}{c}13 \\
0\end{array}$ & 108 & \multirow{4}{*}{ U.3US } & 85 & \multirow{4}{*}{ U.५U/ } & 23 & \multirow{4}{*}{0.31} \\
\hline & II & 41 (53) & \multirow{3}{*}{$\begin{array}{l}50 \\
(54) \\
42 \\
(45) \\
8(y)\end{array}$} & & (4) & & $5 /(13)$ & \\
\hline & ID & $52(6 /)$ & & & $44^{\prime}$ & & $35(8)$ & \\
\hline & UU & $8(10)$ & & & $8(1)$ & & $y(2)$ & \\
\hline & $\nu$ allele & $33(8 /)$ & $\left(\frac{2 y}{63}\right)$ & U.315 & (5) & U.452 & $2 b(12)$ & 0.19 \\
\hline & HVVE & 0.113 & 1.000 & & & & U.6U8 & \\
\hline \multirow{4}{*}{$\begin{array}{l}\text { MIKKouz rs4y1ysiv } \\
\text { (C/G) }\end{array}$} & IV & 12 & 110 & \multirow{4}{*}{ U.145 } & 85 & \multirow{4}{*}{ U.441 } & 25 & \multirow{4}{*}{ U.U46 } \\
\hline & CL & by $(/ 3)$ & \multirow{3}{*}{$\begin{array}{l}(76) \\
(73) \\
(31) \\
6(6)\end{array}$} & & $(64)$ & & $16(19)$ & \\
\hline & LG & $3 y(48)$ & & & (27) & & $16(4)$ & \\
\hline & $G G$ & $2(3)$ & & & $5(4)$ & & $8(2)$ & \\
\hline
\end{tabular}




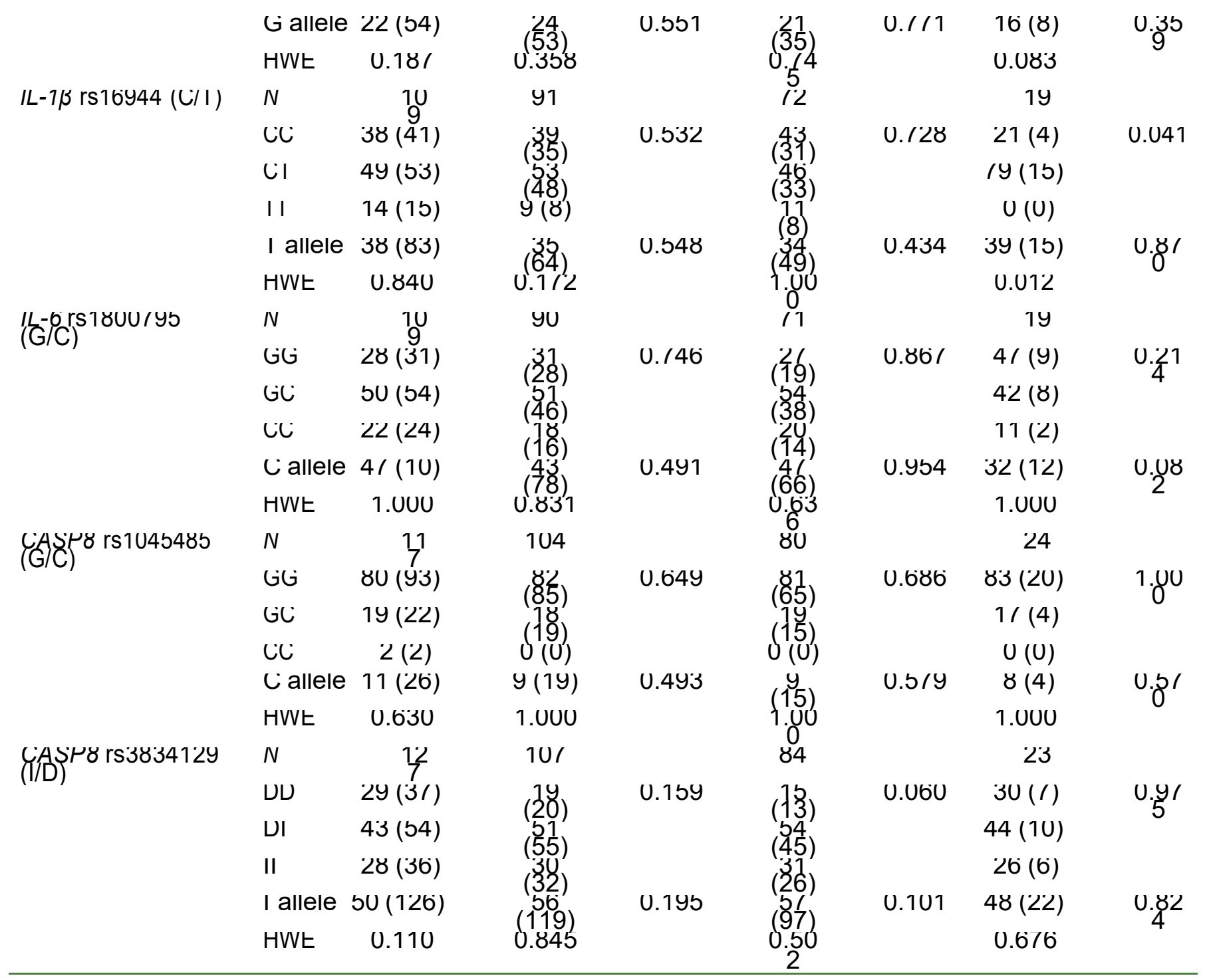

Notes: Genotype and allele frequencies are expressed as percentages with the number of participants shown in parenthesis $(n)$. Global $P$-values are given with boldtypeset indicating significant differences $(P<0.05)$.

$\mathrm{I}$ : insertion allele, $\mathrm{D}$ : deletion allele.

${ }^{\mathrm{a} C O N}$ vs.

ATP.

${ }^{\mathrm{b}} \mathrm{CON}$ vs.

TEN.

${ }^{\mathrm{C} C O N}$ vs.

RUP.

model: $P=0.053$ ). More specifically, the $T-C-D$ combination was found to increase the risk of ATP (additive and dominant models: $P=0.023$ ) and RUP (additive and dominant models: $P<0.000$ ) while the $C-A-I$ combination was found to increase the risk of ATP (additive model: $P=0.011$ ), TEN (dominant model: $P=$ 0.011 ) and RUP (additive model: $P=0.011$ ). Alternatively, the $C-C-D$ combination was associated with a decreased risk of ATP (dominant model: $P=0.011$ ) and RUP (dominant: $P=0.004$ ).

Four possible inferred allele combinations were constructed from COL5A1 rs3196378 and MIR608 rs4919510

[Figure 2(b)]. The A-C inferred allele combination was the most common (overall frequency: $40 \%$ ) whereas the $C-G$ inferred allele combination was the least common (overall frequency: $8 \%$ ). None of the combinations were significantly associated with the risk of ATP.

Inferred allele combinations were constructed from IL-1 $\beta$ rs16944, IL-6 rs1800795 and COL5A1 rs12722 
[Figure 2(c)]. Eight possible combinations that were all similar in range were noted. These eight combinations were equally distributed amongst the cases and controls with no combinations significantly associated with the risk of ATP.

(a)

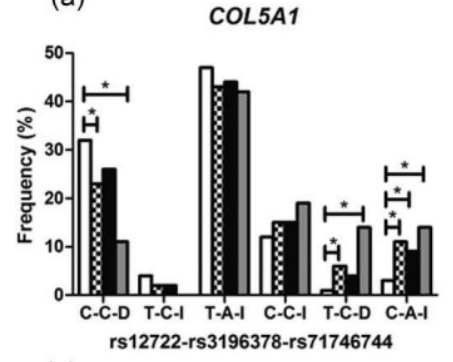

(c)

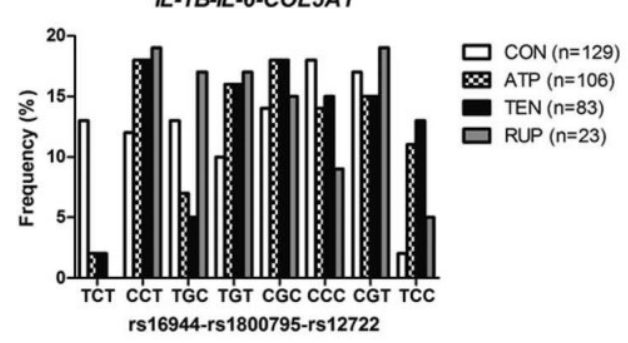

(b)
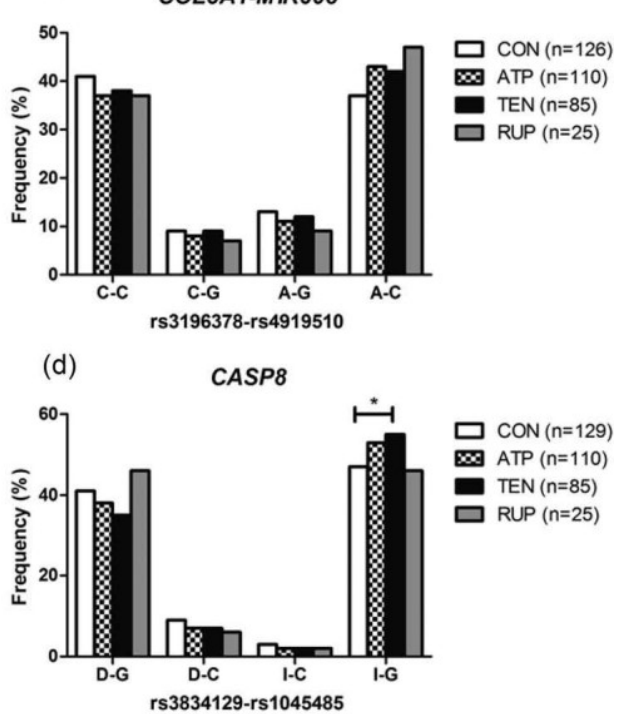

Figure 2. Frequency distributions of the inferred allele combinations for: (a) COL5A1 rs12722, rs3196378 and rs71746744, (b) COL5A1 rs3196378 and MIR608 rs4919510, (c) IL-1ß rs16944, IL-6 rs1800795 and COL5A1 rs12722 and (d) CASP8 rs3834129 and rs1045485 in the CON group (white bars), ATP group (checked bars), TEN sub-group (black bars) and RUP sub-group (grey bars). An asterisk indicates a significant difference between groups $(P<0.05)$. The number of individuals in each group is shown in parenthesis $(n)$.

Four possible inferred allele combinations were constructed from CASP8 rs3834129 and rs1045485 [Figure 2(d)]. D-G and I-G were identified as the two major combinations while D-C and I-C were the two minor combinations. I-G was the most common inferred allele combination (overall frequency: $50 \%$ ) whereas I-C was the least common (overall frequency: $3 \%$ ). Global P-values were not significant for any of the groups; however, considering the a priori hypothesis, significance was observed when P-values for the individual inferred allele combinations were analysed. The I-G combination was significantly associated with an increased risk of TEN (dominant model $P=0.031$ ).

\section{Discussion}

ATP is a multifactorial condition for which a genetic component has been identified (Collins et al., 2015). To date, several specific loci have been associated with risk in independent populations of South African and Australian ancestry. As such, the aim of this study was to evaluate eight previously identified predisposing genetic variants in a third population. Overall, the findings from this association study further support the hypothesis that genetic sequences influencing regulation of the synthesis and degradation of the ECM of tendons may play an important role in modulating risk of ATP. The main findings of this study included (i) the associations of the COL5A1 inferred allele combinations with the risk of all three phenotypes and (ii) the independent and collective associations of CASP8 with the risk of TEN. In addition, MIR608 and IL1B were independently associated with the risk of RUPs, however, as previously highlighted these findings should be interpreted with caution.

The COL5A1 3'UTR has been extensively studied in the aetiology of ATP with multiple sequence variants both independently and collectively noted as risk modifiers (Abrahams et al., 2013; Mokone et al., 2006; September et al., 2009). The inferred allele combinations identified in this study were constructed from three variants situated within the COL5A1 3'UTR. Our findings were in partial agreement with published literature 
and our a priori hypothesis. The T-C (COL5A1 rs12722- rs3196378) inferred allele combination and the COL5A1 rs71746744 II genotype were previously associated with an increased risk of TEN (Abrahams et al., 2013; September et al., 2009). The a priori hypothesis thus included that the T-C-I inferred allele combination increases the risk of ATP in the British cohort. In the current study, three risk modifying inferred allele combinations were detected. Specifically, the T-C-D and C-A-I combinations were associated with an increased risk of ATP while the C-C-D combination was associated with a decreased risk of ATP. Interestingly, the three alleles in the C-C-D inferred allele combination were identified in the major functional form of the COL5A1 3'UTR previously proposed to be associated with reduced risk of chronic TEN (Collins \& Posthumus, 2011; Laguette, Abrahams, Prince, \& Collins, 2011). To date, no study has analysed COL5A1 rs127277, rs3196378 and rs71746744 in an inferred allele combination, hence further analyses in additional independent populations are recommended. Nonetheless, this study was able to further implicate variants within the COL5A1 3'UTR in the aetiology of ATP in a third study group, composed of participants of self-reported British ancestry.

Biologically, polymorphisms within the 3'UTR could affect the 3'UTR secondary structure (Abrahams et al., 2013). This may cause alterations in COL5A1 mRNA stability and subsequently the production of the $\alpha 1$ chain of type $\mathrm{V}$ collagen ( $\alpha 1(\mathrm{~V})$ ) (Abrahams et al., 2013). We have previously identified two functional forms of the COL5A1 3'UTR, namely the Cand T-functional forms, with the C-functional form having decreased mRNA stability compared to the T-form (Laguette et al., 2011). Based on its function and the previous association

of variants within the 3'UTR with altered risk of TEN, the C-functional form has been proposed to reduce the risk of TEN (Collins \& Posthumus, 2011). As previously mentioned, the three alleles in the C-C-D inferred combination which associated with reduced ATP risk in this study were identified in the C-functional form of the COL5A1 3'UTR. Moreover, COL5A1 rs3196378 is located within a Hsa-miR-608 binding site, which Laguette et al. have shown to be functional (Laguette et al., 2011). Since microRNA molecules are involved in gene regulation (He \& Hannon, 2004), differential miRNA binding may also influence the production of a1(V) (Abrahams et al., 2013). These small changes in COL5A1 translation may lead to interindividual variation in fibrillogenesis and predisposition to ATP (Collins \& Posthumus, 2011).

The second main finding of this study implicated CASP8. Individuals with a CASP8 rs3834129 DD genotype were at a 55\% reduced risk of developing TEN. In addition, individuals with the CASP8 I-G (rs3834129rs1045485) inferred allele combination were at an increased risk of developing TEN. Both of these findings are in line with the a priori hypothesis. Nell et al. have reported that the CASP8 rs3834129 DD genotype increases the risk of TEN whereas the ID genotype decreases the risk of TEN (Nell et al., 2012). Nell et al. also found that the D-C inferred allele combination decreases the risk of TEN (Nell et al., 2012). The independent genotype association found in this study is in contrast to the findings of Nell et al. but makes biological sense.

The D allele of CASP8 rs3834129 causes destruction of an Sp1 binding element, resulting in decreased gene expression and reduced levels of caspase-8 protein (Sun et al., 2007). This could prevent excessive apoptosis and protect against TEN (Nell et al., 2012). The inferred allele combination association found in this study is in agreement with previous findings and is also biologically plausible. Since the $D$ allele of CASP8 rs384129 is protective, the I allele is expected to be deleterious. Furthermore, the $G$ allele of CASP8 rs1045485 encodes the wild-type Asp amino acid which is predicted to result in a more stable form of the caspase-8 protein (MacPherson et al., 2004). This promotes apoptosis and may increase the risk of TEN (Nell et al., 2012). Rickaby et al. recently showed that a CASP8 copy number variant, spanning intron 11intron 12, which overlaps CASP8 rs1045485 and is approximately 50000bp from rs3834129, is not associated with ATP in the same British cohort (Rickaby et al., 2015). They subsequently speculated that associations of the CASP8 locus with ATP may be population specific and recommended that the CASP8 variants investigated by Nell et al. (2012) were tested in a British cohort. The current study evaluated these previously studied variants and revealed that the CASP8 locus is also associated with the risk of ATP in an independent British population. However, more samples are required to be investigated at this locus to allow for conclusive evidence. 
The additional findings concerning MIR608 rs4919510 and IL1B rs16944 suggested that (i) individuals with a MIR608 rs4919510 CG genotype were at a 70\% reduced risk of developing RUPs compared to individuals with CC or GG genotypes and (ii) individuals with an IL-1B rs16944 CT genotype were 3.96 times more likely to develop RUPs than individuals with CC or TT genotypes. These findings are novel since no study has found associations between these SNPs and the risk of only RUPs, however, because the RUP subgroup was small, replication in larger study participant groups is crucial for added credibility.

This study was not able to replicate the independent associations of COL5A1 rs12722, COL5A1 rs3196378, COL5A1 rs71746744 or MIR608 rs4919510 with the risk of TEN. Nor were we able to replicate the collective associations of COL5AI-MIR608 or IL-6-IL-1B-COL5A1 with the risk of TEN. This may be reflective of the study limitations. The main limitation was the small sample size, resulting in reduced power when analysing the TEN and RUP sub-groups separately. As such, the findings pertaining to these groups need to be interpreted cautiously, particularly regarding the RUP sub-group $(n=25)$. Second, statistical analyses were conducted without adjusting for possible confounding variables. This presented a limitation when the CON group and TEN sub-group were analysed because participants in the CON group were significantly younger than participants in the TEN sub-group. A third potential limitation was the COL5A1 rs12722 and IL-6 rs1800795 genotype-BMI and genotype- sex associations, respectively. Similar genetic studies in larger cohorts did not, however, report any genotype-BMl or genotype-sex associations for these variants (Mokone et al., 2006; September et al., 2009, 2011). In view of the fact that this study is influenced by the small sample size; to fully comprehend the involvement of these loci in the aetiology of ATP, it is recommended that this study is repeated in a larger study group. Finally, the different pathology phenotypes in the TEN and RUP sub-groups was a potential limitation. The TEN sub-group consisted of individuals diagnosed with insertional and non-insertional tendinopathy while the RUP sub-group consisted of individuals diagnosed with complete and partial ruptures. Furthermore, combining the TEN and RUP subgroups into the ATP group resulted in the grouping of chronic and acute pathologies. The possibility of different injury mechanisms in these groups cannot be excluded.

The major difference between the current study and previous studies is the investigation of these eight variants in an association study with RUP risk. There is evidence to suggest that the incidence of acute RUPs is increasing and the exact mechanism underlying this increase remains to be explored (Huttunen, Kannus, Rolf, Fellander-Tsai, \& Mattila, 2014; Lantto, Heikkinen, Flinkkilä, Ohtonen, \& Leppilahti, 2015). To increase our understanding of these eight functional variants and the risk of ATP, it is important that they are investigated in chronic and acute injuries. It is, therefore, necessary to understand the differences between the incidences of ATP in various populations. However, there is currently limited published epidemiological data for effective comparison.

In conclusion, this study further highlights the importance of polymorphisms within the COL5A1 and CASP8 genes in the aetiology of ATP. In addition, the potential role of the MIR608 and IL-1 $\beta$ variants was reiterated but only within the small RUP sub-group. It would, therefore, be beneficial to repeat this genetic association study in a larger British cohort. Finally, genetic sequencing will play a pivotal role in expediting the identification of sequence variants which affect susceptibility to ATP and will contribute to broadening our understanding of injury pathogenesis.

Acknowledgements

K.L.B. and K.B.S. were funded by the National Research Foundation (NRF) of South Africa. M.P. was funded by the Thembakazi Trust.

Disclosure statement

No potential conflict of interest was reported by the authors.

Funding

This work was supported by the National Research Foundation (NRF) of South Africa and Thembakazi Trust. 


\section{References}

Abrahams, Y., Laguette, M., Prince, S., \& Collins, M. (2013). Polymorphisms within the COL5A1 3'-UTR that alters mRNA structure and the MIR608 gene are associated with Achilles tendinopathy. Annals of Human Genetics, 77, 204-214. doi:10.1111/ahg.12013

Birkedal-Hansen, H., Moore, W. G. I., Bodden, M. K., Windsor, L. J., Decarlo, A., \& Engler, J. A. (1993). Matrix metalloproteinases: A review. Nature Reviews Molecular Cell Biology, 4(2), 197-250.

doi:10.1038/nrm2125

Chanock, S., Manolio, T., Boehnke, M., Boerwinkle, E., Hunter, D. J., Thomas, G., . . Collins, F. S. (2007). Replicating genotype-phenotype associations. Nature, 447(7145), 655-660. doi:10.1038/447655a

Collins, M., \& Posthumus, M. (2011). Type V collagen genotype and exercise-related phenotype relationships: A novel hypothesis. Exercise and Sport Sciences Reviews, 39(4), 191-198. doi:10.1097/ JES.0b013e318224e853

Collins, M., September, A. V., \& Posthumus, M. (2015). Biological variation in musculoskeletal injuries: Current knowledge, future research and practical implications. British Journal of Sports Medicine, 1-8. doi:10.1136/bjsports-2015-095180

Fain, J. N. (2006). Release of interleukins and other inflammatory cytokines by human adipose tissue is enhanced in obesity and primarily due to the nonfat cells. Vitamins and Hormones, 74, 443-477.

doi:10.1016/ S0083-6729(06)74018-3

Gaida, J. E., Ashe, M. C., Bass, S. L., \& Cook, J. L. (2009). Is adiposity an under-recognized risk factor for tendinopathy? A systematic review. Arthritis \& Rheumatism, 61(6), 840-849. doi:10.1002/art.24518

González, J., Armengol, L., Solé, X., Guino, E., Mercader, J., \& Esyivill, X. (2007). SNPassoc: An R package to perform whole genome association studies. Bioinformatics, 23(5), 654-655.

doi:10.1093/bioinformatics/btm025

Haskell, W. L., Lee, I., Pate, R. R., Powell, K. E., Blair, S. N., Franklin, B. A., . . Bauman, A. (2007). Physical activity and public health updated recommendation for adults from the American college of sports medicine and the american heart association. Circulation, 116(9), 1081-1094.

doi:10.1161/CIRCULATIONAHA.107.185649

He, L., \& Hannon, G. J. (2004). MicroRNAs: Small RNAs with a big role in gene regulation. Nature Reviews. Genetics, 5(7), 522-531. doi:10.1038/ nrg1379

Huttunen, T. T., Kannus, P., Rolf, C., Fellander-Tsai, L., \& Mattila, V. M. (2014). Acute achilles tendon ruptures: Incidence of injury and surgery in Sweden between 2001 and 2012. The American Journal of Sports Medicine, 42(10), 2419-2423. doi:10.1177/0363546514540599

Järvinen, T. A. H., Kannus, P., Maffulli, N., \& Khan, K. M. (2005). Achilles tendon disorders: Etiology and epidemiology. Foot and Ankle Clinics, 10 (2), 255-266. doi:10.1016/j.fcl.2005.01.013

Kahai, S., Vary, C. P. H., Gao, Y., \& Seth, A. (2004). Collagen, type V, $\alpha 1$ (COL5A1) is regulated by TGF- $\beta$ in osteoblasts. Matrix Biology, 23(7), 445-455. doi:10.1016/j.matbio.2004.09.004

Laguette, M.-J., Abrahams, Y., Prince, S., \& Collins, M. (2011). Sequence variants within the 3'-UTR of the COL5A1 gene alters mRNA stability: Implications for musculoskeletal soft tissue injuries. Matrix Biology, 30 (5-6), 338-345. doi:10.1016/j.matbio.2011.05.001 
Lantto, I., Heikkinen, J., Flinkkilä, T., Ohtonen, P., \& Leppilahti, J. (2015). Epidemiology of Achilles tendon ruptures: Increasing incidence over a 33-year period. Scandinavian Journal of Medicine \& Science in Sports, 25 (1), e133-e138. doi:10.1111/sms.12253

Little, J., Higgins, J. P. T., loannidis, J. P. A., Moher, D., Gagnon, F., von Elm, E., . . Birkett, N. (2009). STrengthening the REporting of Genetic Association studies (STREGA) An extension of the STROBE statement. European Journal of Clinical Investigation, 39(4), 247-266. doi:10.1111/ j.1365-

2362.2009.02125.x

MacPherson, G., Healey, C. S., Teare, M. D., Balasubramanian, S. P., Reed, M.,W. R., Pharoah, P. D. P., . .. Cox, A. (2004). Association of a common variant of the CASP8 gene with reduced risk of breast cancer. $\mathrm{JNCl}$ Journal of the National Cancer Institute, 96(24), 1866-1869. doi:10.1093/ jnci/dji001

Meeuwisse, W. H. (1994). Assessing causation in sport injury: A multifactorial model. Clinical Journal of Sport Medicine, 4(3), 166-170. doi:10.1097/00042752-199407000-00004

Millar, N. L., Wei, A. Q., Molloy, T. J., Bonar, F., \& Murrell, G. A. C. (2009). Cytokines and apoptosis in supraspinatus tendinopathy. The Journal of Bone and Joint Surgery. British Volume, 91-B(3), 417-424. doi:10.1302/ 0301-620X.91B3.21652

Mokone, G. G., Gajjar, M., September, A. V., Schwellnus, M. P., Greenberg, J., Noakes, T. D., \& Collins, M. (2005). The guanine-thymine dinucleotide repeat polymorphism within the tenascin-C gene is associated with achilles tendon injuries. The American Journal of Sports Medicine, 33 (7), 1016-1021.

doi:10.1177/0363546504271986

Mokone, G. G., Schwellnus, M. P., Noakes, T. D., \& Collins, M. (2006). The COL5A1 gene and Achilles tendon pathology. Scandinavian Journal of Medicine \& Science in Sports, 16(1), 19-26. doi:10.1111/j.16000838.2005.00439.x

Nell, E., Van Der Merwe, L., Cook, J., Handley, C. J., Collins, M., \& September, A. V. (2012). The apoptosis pathway and the genetic predisposition to Achilles tendinopathy. Journal of Orthopaedic Research Month, 1-6. doi:10.1002/jor.22144

Newnham, D. M., Douglas, J. G., Legge, J. S., \& Friend, J. A. R. (1991). Achilles tendon rupture: An underrated complication of corticosteroid treatment. Thorax, 46(11), 853-854. doi:10.1136/thx.46.11.853

R Development Core Team. (2013). R: A language and environment for statistical computing. Vienna: R Foundation for Statistical Computing. Retrieved from http://www.r-project.org/

Rickaby, R., El Khoury, L., Ribbans, W. J., \& Raleigh, S. M. (2015). Variation within three apoptosis associated genes as potential risk factors for Achilles tendinopathy in a British based case-control cohort. Gene, 571 (2), 167-171. doi:10.1016/j.gene.2015.06.010

Riley, G. P. (2005). Gene expression and matrix turnover in overused and damaged tendons. Scandinavian Journal of Medicine \& Science in Sports, 15(4), 241-251. doi:10.1111/j.1600-0838.2005.00456.x

Scott, A., Backman, L. J., \& Speed, C. (2015). Tendinopathy: Update on pathophysiology. Journal of Orthopaedic \& Sports Physical Therapy, 45 (11), 833-841. doi:10.2519/jospt.2015.5884

September, A. V., Cook, J., Handley, C. J., Van Der Merwe, L., Schwellnus, M. P., \& Collins, M. (2009). Variants within the COL5A1 gene are associated with Achilles tendinopathy in two populations. British Journal of Sports Medicine, 43, 357-365. doi:10.1136/bjsm.2008.048793

September, A. V., Nell, E.-M., O'Connell, K., Cook, J., Handley, C. J., van der Merwe, L., Collins, M. (2011). A pathway-based approach investigating the genes encoding interleukin- $1 \beta$, interleukin- 6 and the 
interleukin-1 receptor antagonist provides new insight into the genetic susceptibility of Achilles tendinopathy. British Journal of Sports Medicine, 45(13), 1040-1047. doi:10.1136/bjsm.2010.076760

Sinnwell, J. P., \& Schaid, D. J. (2008). Haplo stats statistical methods for haplotypes when linkage phase is ambiguous. R package Version 1.4.4. Retrieved from http://cran.r-project.org/package=haplo.stats

Strandberg, L., Mellström, D., Ljunggren, Ö., Grundberg, E., Karlsson, M. K., Holmberg, A. H., . . Jansson, J.-O. (2008). IL6 and IL1B polymorphisms are associated with fat mass in older men: The MrOS Study Sweden. Obesity (Silver Spring, Md.), 16(3), 710-713. doi:10.1038/ oby.2007.95

Sun, H. B., Li, Y., Fung, D. T., Majeska, R. J., Schaffler, M. B., \& Flatow, E. L. (2008). Coordinate regulation of IL-1 $\beta$ and MMP-13 in rat tendons following subrupture fatigue damage. Clinical Orthopaedics and Related Research, 466(7), 1555-1561. doi:10.1007/s11999-008-0278-4

Sun, T., Gao, Y., Tan, W., Ma, S., Shi, Y., Yao, J., . . Lin, D. (2007). A sixnucleotide insertion-deletion polymorphism in the CASP8 promoter is associated with susceptibility to multiple cancers. Nature Genetics, 39, 605-613. doi:10.1038/ng2030

Thampattya, B., Li, H., Im, H., \& Wang, J. (2007). EP4 receptor regulates collagen type-I, MMP-1, and MMP-3 gene expression in human tendon fibroblasts in response to IL-1 $\beta$ treatment. Gene, 386(1-2), 154161. doi:10.1016/j.gene.2006.08.027

Tsuzaki, M., Guyton, G., Garrett, W., Archambault, J. M., Herzog, W., Almekinders, L., .Banes, A. J. (2003). IL-1 $\beta$ induces COX2, MMP-1, -3 and -13 , ADAMTS-4, IL-1 $\beta$ and IL-6 in human tendon cells. Journal of Orthopaedic Research, 21, 256-264. doi:10.1016/S0736-0266(02)00141-9 Van der Linden, P. D., Nab, H. W., Simonian, S., Stricker, B. H. C., Leufkens,

H. G. M., \& Herings, R. M. C. (2001). Fluoroquinolone use and the change in incidence of tendon ruptures in the Netherlands. Pharmacy World and Science, 23(3), 89-92. doi:10.1023/A:1011254030271

Villiger, P. M., Kusari, A. B., ten Dijke, P., \& Lotz, M. (1993). IL-1 beta and IL-6 selectively induce transforming growth factor-beta isoforms in human articular chondrocytes. Journal of Immunology, 151(6), 3337-3344.

Von Elm, E., Altman, D. G., Egger, M., Pocock, S. J., Gøtzsche, C., \& Vandenbroucke, J. P. (2007). Policy and practice the strengthening the reporting of observational studies in epidemiology (STROBE) statement: Guidelines for reporting observational studies. Bulletin of the World Health Organization, 85(11), 867-872. doi:10.2471/BLT.07.045120

Wenstrup, R. J., Florer, J. B., Brunskill, E. W., Bell, S. M., Chervoneva, I., \& Birk, D. E. (2004). Type V collagen controls the initiation of collagen fibril assembly. The Journal of Biological Chemistry, 279(51), 53331-53337. doi:10.1074/jbc.M409622200

Yuan, J., Murrell, G. A. C., Wei, A.-Q., \& Wang, M.-X. (2002). Apoptosis in rotator cuff tendonopathy. Journal of Orthopaedic Research: Official Publication of the Orthopaedic Research Society, 20(6), 13721379. doi:10.1016/S0736-0266(02)00075-X 